\title{
ON CONSTITUTIVE EQUATIONS FOR BRANCHING OF RESPONSE WITH SELECTIVITY
}

\author{
K. R. Rajagopal and A. Wineman. \\ Department of Applied Mechanics and Engineering Science, The University of Michigan, \\ Ann Arbor, MI 48109, U.S.A.
}

(Received 12 March 1979)

\begin{abstract}
Materials, such as elastic-plastic, which exhibit distinct regimes of response are usually modeled by different constitutive equations in each regime. The present paper explores a method for the construction of a unified constitutive equation from these separate relations. The main idea is to write this unified equation in an implicit form which contains these separate solutions as nonunique solutions. The form is chosen in order to utilize the notions of branch points and branches. Different solutions, corresponding to constitutive equations for different regimes of response, are then regarded as bifurcations at branch points from the fundamental response. The choice of the appropriate branch at a branch point is governed by a selectivity condition which depends on the nature of the response under consideration. A detailed example is provided for elastic-plastic response, with and without the effect of strain rate dependence.
\end{abstract}

\section{INTRODUCTION}

Most of the constitutive equations which have been developed express the stress in terms of the history of the deformation gradient using operators which are in some sense very smooth. That is, in models for elastic and viscoelastic response, the operators are assumed to be at least differentiable. An important exception is the constitutive equation for elasticplastic response. Here, one expression is given for the elastic range and another for the plastic range, along with a conditionality statement for switching from one to the other. The expressions for the two ranges can be interpreted, in a sense to be described more fully later, as branches of a single constitutive equation. Thus while the stress-strain relations are continuous they are not differentiable everywhere, namely where the branching takes place.

There are other phenomena for which the constitutive operator may consist of several branches. One example would be provided by a uniaxial stress-strain graph with kinks. This could represent the response of a matrix embedded with slack fibers. The initial part of the curve represents the response of the matrix. The kink is produced when the fibers are sufficiently extended so that they offer resistance. Another example would be associated with a uniaxial instability observed in polyethylene filaments. At a certain strain, the filament undergoes a sudden reduction in diameter and becomes stiffer. The pre- and postnecked states have been regarded by Zapas [1] and Ericksen [2] as changes in material phase. Separate stress-strain relations apply to each phase.

There have been studies of constitutive equations which incorporate branches into a single expression. The essential mathematical device is to write the constitutive equation in implicit form. This introduces a non-linear equation whose solution for stress in terms of strain is not unique. Morgan [3] considered equations of the form $f(\sigma, \varepsilon)=0$ where $f$ is an isotropic polynomial in two matrix arguments. The branches arise through a failure of the condition necessary for the stress to be found uniquely in terms of the strain. However, this study was confined to assumed or general forms of the implicit constitutive equation. It would be more desirable to have a constructive approach in which the various branches are combined into a single constitutive equation. One may then use this approach to model material behavior in the light of experimental results. One method of achieving this was presented by Bernstein [4] in a unified constitutive equation for elastic-plastic response. The model relates non-linear differential operators on stress and strain. The Lipshitz condition for uniqueness of the solution to this equation for stress in terms of strain fails to hold when the yield condition is satisfied. There are two solutions, one for elastic unloading and one for which the yield condition continues to be met. When unloading is to occur, 
only the elastic solution is stable, while the same holds for the plastic solution during loading. Thus, the model not only has several branches, but also a preference for the branch which is appropriate to the solution.

The purpose of the present work is to explore further the possibility of developing a theory for a unified constitutive equation which is not only capable of modeling branching of response but also of selecting an appropriate branch.

Our point of view is that such a selection process is characteristic of every material which shows distinct regimes of response. That is, for every material there is some range of deformation in which the stress-strain relation has one characteristic shape, say linear, while for larger deformations the stress-strain relation has a distinctly different shape, say non-linear. We interpret this deviatory behavior by the material of branching from one characteristic response to another as a selection process which the material is required to make due to certain a priori restrictions placed on its behavior. These restrictions, of course, have to be physically meaningful. A material is then completely characterized by means of an implicit constitutive equation and an additional 'decision making' constraint. Thus, two different materials which can be classified as elastic-plastic, and which have the same elastic modulus, are distinguished by their 'decision' about when to yield. This 'decision' would, of course, be based on a criterion which is common for materials belonging to the same category and would differ from one category to another.

The ideas presented here are an outgrowth of those of Bernstein [4], and those presented by Stakgold [5] in a review paper on the branching of solutions of nonlinear equations. For the sake of simplicity, attention is confined here to constitutive equations for onedimensional response. Extensions to higher dimensions will follow in the near future.

The essential notions regarding branching are introduced by means of examples in Section 2. A precise mathematical statement is presented in Section 3. An application to elastic-plastic response, including strain rate effects, is given in Section 4.

\section{EXAMPLES OF BRANCHING}

In order to introduce the notions of branching and selectivity, consider the well known uniaxial response of metallic materials which are loaded at constant strain rate. In loading in tension or compression from the undeformed state, there is typically a set of values of $\varepsilon$ for which the stress-strain relation is linear. This is followed by another set of values of $\varepsilon$ for which stress and strain are related by a different expression. The new expression reflects a change in the nature of the processes underlying the response of the material.

One approach to the modeling of this response is to write a relation of the form

$$
\sigma=E \varepsilon+g(\varepsilon),
$$

which is to hold for the complete set of values of $\varepsilon$ under consideration. An example of such an approach is an inverted form of (2.1) known as the Ramberg-Osgood equation [6]. The function $g(\varepsilon)$ is very smooth. Its values are negligible compared to the first term in (2.1) for $|\varepsilon|<\alpha$. They become large only for $|\varepsilon|>\alpha$ in order to yield the desired values for $\sigma$. This approach has been used in modeling elastic-plastic response. It depends primarily on one's cleverness in constructing a satisfactory function $g(\varepsilon)$.

As an alternate approach, consider an implicit relation between stress and strain, $F(\sigma, \varepsilon)=0$, of the form

$$
F(\sigma, \varepsilon)=\left[(\sigma-E b)^{2}-(\varepsilon-b)\right]\left[(\sigma+E b)^{2}+(\varepsilon+b)\right][\sigma-E \varepsilon]=0 .
$$

The pairs $(\sigma, \varepsilon)$ which satisfy this relation are shown in Fig. 1.

Starting from the unstretched state, suppose $\varepsilon$ is increased until $\varepsilon=b$. Since the first two factors in $F(\sigma, \varepsilon)$ in (2.2) do not have real solutions in this range, the stress-strain curve is given by the last factor. This appears as curve $0 A$. When $\varepsilon$ is increased further, it is possible that the stress-strain curve could be any one of the three curves shown. However, the material 'selects' one of these three available responses, say $A 1$, due to a criterion based on certain restrictions which are placed on material behavior. With appropriate restrictions, one can obtain the usual stress-strain curve from the choices shown in Fig. 2. Within this 


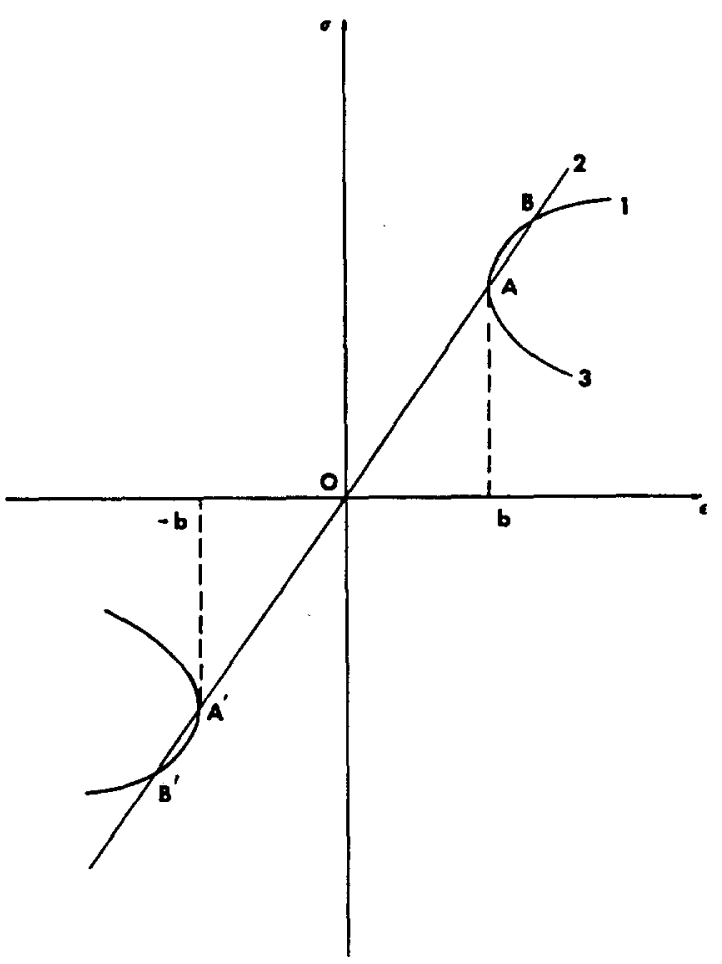

Fig. 1. Locus of the equation (2.2), showing the branches.

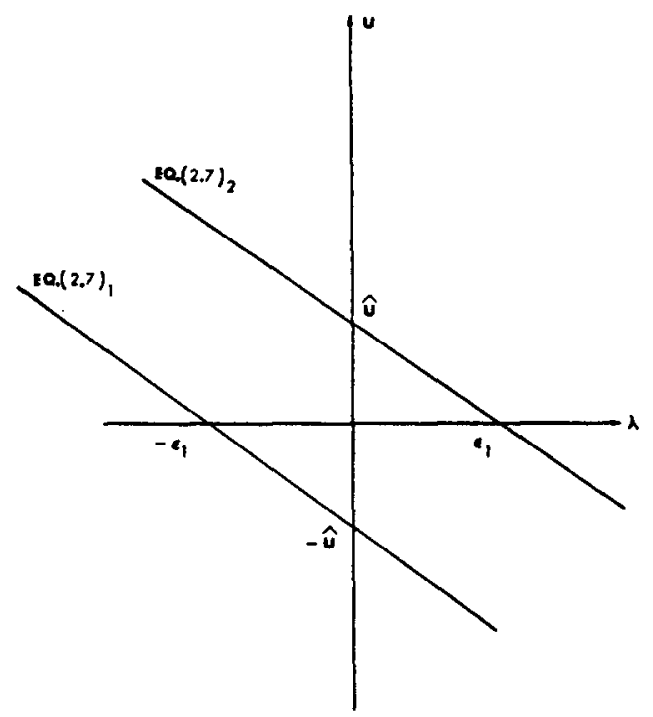

Fig. 2. The graphs of the solutions of equation $(2.6)$ in the $(u, \lambda)$ plane.

category of material response, it is seen that different materials can be modeled by selecting different values for $b$ and $E$ and different coefficients in (2.2).

A point such as $A$ in Fig. 1 will be called a branch point and the possible response curves passing through $A$ will be called branches. This terminology is deliberate, since the problem of modeling the response is viewed, not as one of determining an analytic correction as suggested by (2.1), but as a branching problem in mathematics. In fact, the above example is a special case of the more general stress-strain relation

$$
F(\sigma, \varepsilon)=L(u) f(\sigma, \varepsilon)=0,
$$

where $u=\sigma-E \varepsilon$ and $L$ is a linear operator. It follows that $u=0$ is a solution of (2.3) and thus 
one of the branches is the linear elastic response. This shall be considered the "fundamental state.' Deviations from linear elastic response then correspond to the mathematical problem of the determination of branching from the fundamental state.

Certainly, other forms of implicit stress-strain relations could also be concocted. For many materials, they could be reduced to the form

$$
A u-\lambda(\varepsilon) u=0,
$$

where $u=\sigma-E \varepsilon$. The operator $A$ is, in general, completely continuous with nice smoothness properties at the origin. It is then possible to use the approach in the article by Stakgold [5] to study such materials. The existence of branch points of the basic solution for such operators is related to the multiplicity of the eigenvalues of the linearized operator $L \equiv A^{\prime}(0)$.

While there is no choice of the non-linear operator $A$ for which the preceding example can be put in the form of (2.4), the following example shows that stress-strain relations of this form can be used to model important material response. Consider the piece-wise-linear stress-strain relation for elastic-strain hardening response shown in Fig. 3. Operator $A$ is defined as

$$
A u=\left(\varepsilon_{1}\right)^{2} u-\frac{\left(\varepsilon_{1}\right)^{2}}{\hat{u}^{2}} u^{3}-\frac{\varepsilon_{1} \varepsilon}{\hat{u}} u^{2}-\varepsilon^{2} u+\varepsilon u
$$

where $\varepsilon_{1}$ and $\hat{u}$ are constants such that $\varepsilon_{1}>0$ and $\hat{u}<E \varepsilon_{1}$. Let $\lambda(\varepsilon)=\varepsilon$. Then (2.4), (2.5) reduce to

$$
A u-\lambda(\varepsilon) u=\left(\varepsilon_{1}-\frac{\varepsilon_{1}}{\hat{u}} u-\varepsilon\right)\left(\varepsilon_{1}+\frac{\varepsilon_{1}}{\hat{u}} u+\varepsilon\right) u=0 .
$$

In addition to the null state $u=0$ there are the solutions

$$
u=-\frac{\hat{u}}{\varepsilon_{1}} \varepsilon-\hat{u} \text { and } u=-\frac{\hat{u}}{\varepsilon_{1}} \varepsilon+\hat{u}
$$

The graph of the solutions in the $(u, \lambda)$ plane is shown in Fig. 2 and the $(\sigma, \varepsilon)$ plot is shown in Fig. 3. The branch point is at $\varepsilon=\varepsilon_{1}$. The condition $\hat{u}<E \varepsilon_{1}$ ensures that branch 2 has positive slope, which can be varied by choosing different values of $\hat{u}$.

With these examples in mind, a precise presentation can now be given.

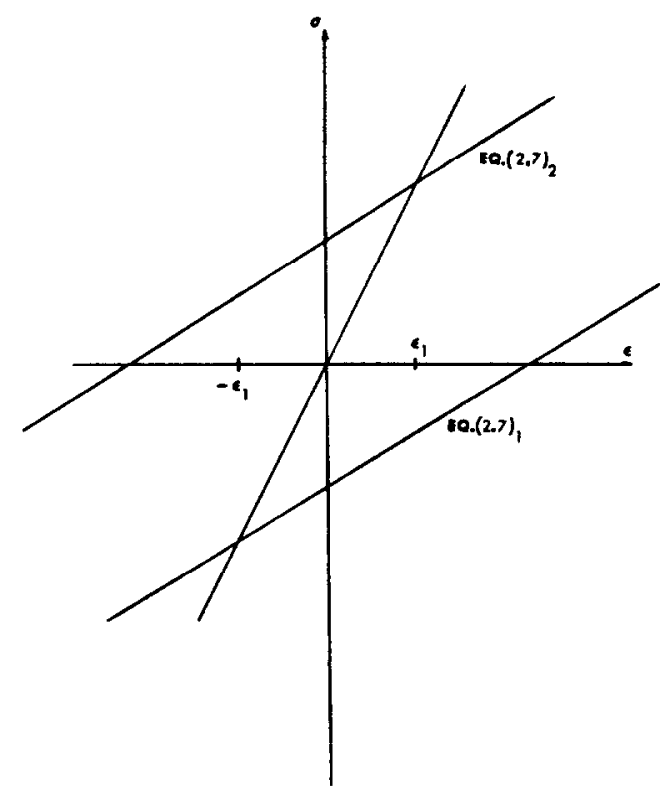

Fig. 3. The graphs of the solutions of equation $(2.6)$ in the $(\sigma, \varepsilon)$ plane. 


\section{MATHEMATICAL FORMULATION}

For convenience, the following definitions refer to stress-strain equations of form (2.3). Their modification for other forms, such as $(2.4)$ is straightforward. Let $\mathscr{R}$ denote the real numbers.

\section{Definition 1 (branch point, branches)}

A point $\left(\sigma_{0}, \varepsilon_{0}\right)$ is called a branch point of $(2.3)$ if in every neighborhood of $\left(\sigma_{0}, \varepsilon_{0}\right) \in \mathscr{R} \times \mathscr{R}$ there exists a solution $(\sigma, \varepsilon)$ of (2.3) such that $\sigma-E \varepsilon \neq 0$. If there exist closed balls $C_{i} \subset R$ which contain $\varepsilon_{0}$, and differentiable functions $g_{i}: C_{i} \rightarrow \mathscr{R}$ such that $\left(g_{i}(\varepsilon), \varepsilon\right)$ solves (2.3), we say that $g_{i}(\varepsilon)$ is a branch at $\varepsilon_{0}$.

Thus in Fig. 1, while A1, A2, A3 would qualify to be branches at A, OA1 would not qualify to be a branch, because it does not meet the differentiability requirements of bring a branch.

Attention will be confined to relations $F$ in (2.3) which lead to only finitely many branches at each branch point. Also implicit relations (2.3), which at any branch point give rise to more than one branch with a particular slope, will not be considered.

As the examples of the preceding section show, the physical stress-strain equation is a subset of points constructed from the mathematical branches. A rational method of obtaining this subset depends on the notion of selectivity discussed earlier. It is assumed that there is a natural selection process for each class of material behavior. Its specification is part of the modeling process and is based on an understanding of the mechanisms underlying the observed mechanical response and the mathematical form of the branches representing these processes. The selectivity criterion presented in the following definition appears to be appropriate for a large class of material response, including elastic-plastic behavior.

\section{Definition 2 (response function)}

A function $\phi: \mathscr{R} \rightarrow \mathscr{R}$ is said to be a response function only if (1) [ $\phi(\varepsilon), \varepsilon]$ satisfies $(2.3)$; (2) $d \phi / d \varepsilon \geqslant 0$; (3) (Selectivity condition) at any point $\left(\phi\left(\varepsilon_{0}\right), \varepsilon_{0}\right)$ which is a branch point, $\phi(\varepsilon)$ is such that

$$
\phi(\varepsilon)=\left\{\begin{array}{lll}
g_{L}(\varepsilon) & \text { if } \quad \phi\left(\varepsilon_{0}\right) \mathrm{d} \varepsilon \geqslant 0 \\
g_{v}(\varepsilon) & \text { if } \quad \phi\left(\varepsilon_{0}\right) \mathrm{d} \varepsilon<0
\end{array}\right.
$$

where $g_{L}$ and $g_{U}$ are branches at $\left(\phi\left(\varepsilon_{0}\right), \varepsilon_{0}\right)$ such that*

$$
\begin{aligned}
& \frac{\mathrm{d} g_{L}}{\mathrm{~d} \varepsilon} \leqslant \frac{\mathrm{d} g_{i}}{\mathrm{~d} \varepsilon}, \quad i=1,2, \ldots, n, \\
& \frac{\mathrm{d} g_{U}}{\mathrm{~d} \varepsilon} \geqslant \frac{\mathrm{d} g_{i}}{\mathrm{~d} \varepsilon}, \quad i=1,2, \ldots, n .
\end{aligned}
$$

Restriction (2) is based on the experimental observation, and the expectation based on thermodynamics, that the increments in stress and strain have the same sign during loading or unloading. Restriction (3) expresses the selectivity condition. At any branch point, the response function is selected from among the branches in order to minimize the increment in expended energy on loading, or to maximize the increment of recovered energy on unloading. In this context, $g_{L}$ and $g_{U}$ represent the loading and unloading response functions, respectively.

There is a class of material behavior for which the above selectivity criterion is definitely not appropriate. Consider the example, referred to in the introduction, of a matrix embedded with slack fibers. The stress-strain relation passing through the origin represents the effect of only the matrix and has a relatively small slope. This is intersected by a steeper branch at the strain where the slack in the fibers is removed. The increased slope represents the effect of the added fiber stiffness. A different selectivity criterion is now required, perhaps

\footnotetext{
*Our assumption that there does not exist more than one branch with a particular slope assures us that the branches $g_{L}$ and $g_{v}$ are well defined.
} 
one based on the concept of maximizing the stiffness. This will not be pursued any further in this paper, and attention will be confined to the former criterion.

\section{ELASTIC-PLASTIC MATERIALS}

The concepts just introduced are now applied to the development of a constitutive equation for elastic-plastic behavior, including the effect of strain-hardening. Strain rate independent response is considered first. The incorporation of strain rate effects will be discussed at the end of the section.

Elastic-plastic response can be characterized by an implicit constitutive equation of form (2.3). Let $L$ and $f(\sigma, \varepsilon)$ be defined by

$$
\begin{gathered}
L=\mathrm{d} / \mathrm{d} t \\
f(\sigma, \varepsilon)=(\sigma-\lambda \varepsilon+k)(\sigma-\lambda \varepsilon-k),
\end{gathered}
$$

where $\lambda$ and $k$ are constants with $0 \leqslant \lambda<E$. Equation (2.3) then becomes

$$
\left[\frac{\mathrm{d}}{\mathrm{d} t}(\sigma-E \varepsilon)\right](\sigma-\lambda \varepsilon+k)(\sigma-\lambda \varepsilon-k)=0 \text {. }
$$

Clearly, a relation between $\sigma$ and $\varepsilon$ such that

$$
\frac{\mathrm{d}}{\mathrm{d} t}(\sigma-E \varepsilon)=0
$$

or

$$
\sigma-\lambda \varepsilon-k=0
$$

or

$$
\sigma-\lambda \varepsilon+k=0,
$$

will meet the requirements of (4.2). We find that the solution (4.3) represents elastic loading or unloading, depending on the initial conditions. The solutions $(4.3)_{2,3}$ represent plastic response, where $k$ is the yield stress and $\lambda$ measures the amount of strain hardening. If $\lambda=0$, we find $(4.3)_{2,3}$ represent the plastic yielding corresponding to an elastic-perfectly plastic material.

Let us consider the response to loading from the initial state $(0,0)$ of a material modeled by the implicit constitutive form (4.1). Then, referring to Fig. 4 , as the strain $\varepsilon$ increases, the response curve follows $0 A$, (solution $(4.3)_{1}$ ), until it reaches the branch point $A$ where the

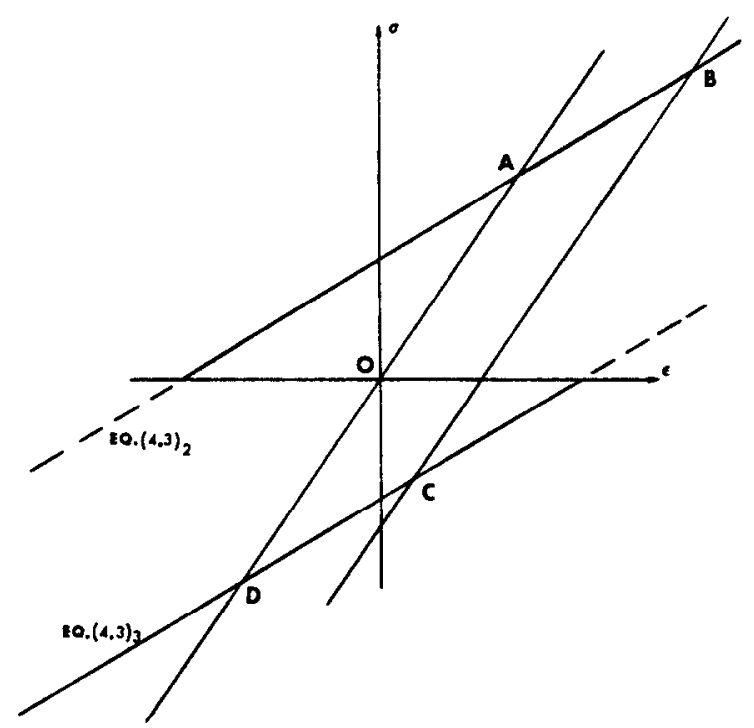

Fig. 4. Branches of equation (4.2) for the elastic-plastic model with no strain rate dependence. 
strain is $\varepsilon_{0}=k /(E-\lambda)$. When the strain $\varepsilon$ is increased further, the response follows branch $A B$ by virtue of the selectivity condition. Suppose the loading is stopped at point $B,\left(\sigma_{B}, \varepsilon_{B}\right)$, and the straining is reversed, i.e. $d \varepsilon<0$. Firstly, we note that any point $(\sigma, \varepsilon)$ which satisfies $(4.3)_{2}\left[\right.$ or $\left.(4.3)_{3}\right]$ is a branch point, the branches satisfying either solutions $(4.3)_{1}$ or $(4.3)_{2}$, [or $(4.3)_{3}$ ]. Since $d \varepsilon<0$, the response could proceed along $B A$ or $B C$, which would then satisfy $(4.3)_{2}$ or $(4.3)_{1}$, respectively. However, due to the selectivity condition, the response proceeds along $B C$ until the branch point $C$ is reached. On further unloading, the response proceeds along $C D$, by virtue of the selectivity condition. Suppose the straining is reversed at point $D$ (i.e. $\mathrm{d} \varepsilon>0$ ). At the branch point $D$, the response could proceed along the branch $D 0$ or $D C$, which respectively meet $(4.3)_{1}$ and $(4.3)_{3}$. The selectivity condition requires that the response proceeds along $D 0$. Continuing in this manner, one can always determine the response of the material based on the condition of selectivity.

Experimental evidence suggest that the values of the strain hardening paramenter $\lambda$ for most materials, and the range of strains in normal applications, are sufficiently small so that the dashed line portions of branches $(4.3)_{2,3}$ shown in Fig. 4 are never encountered. In other words, solutions of $(4.3)_{2}$ with $\sigma<0$ or of $(4.3)_{3}$ with $\sigma>0$ are not expected. If this turns out not to be the case for some materials, then the selectivity condition must be modified. Otherwise, for example, $\phi\left(\varepsilon_{0}\right) d \varepsilon$ could become positive during unloading. This difficulty is avoided by modifying the selectivity condition to be:

Let $\left(\phi\left(\varepsilon_{0}\right), \varepsilon_{0}\right)$ be a branch point. Let $\left(\phi\left(\varepsilon_{0}^{-}\right), \varepsilon_{0}^{-}\right)$be a point on the branch just followed. Let $\left(\phi\left(\varepsilon_{0}^{+}\right), \varepsilon_{0}^{+}\right)$be a point on the branch to be followed next. Then this branch is such that

$$
\phi(\varepsilon)=\left\{\begin{array}{l}
g_{L}(\varepsilon) \\
g_{v}(\varepsilon)
\end{array} \text { if }\left[\phi\left(\varepsilon_{0}\right)-\phi\left(\varepsilon_{0}^{-}\right)\right]\left[\left(\varepsilon_{0}^{+}\right)-\varepsilon_{0}\right]_{<0}^{\geqslant 0},\right.
$$

where $g_{L}$ and $g_{U}$ are branches at $\left(\phi\left(\varepsilon_{0}\right), \varepsilon_{0}\right)$ such that

$$
\begin{aligned}
& \frac{\mathrm{d} g_{L}}{\mathrm{~d} \varepsilon} \leqslant \frac{\mathrm{d} g_{i}}{\mathrm{~d} \varepsilon} \\
& \frac{\mathrm{d} g_{U}}{\mathrm{~d} \varepsilon} \geqslant \frac{\mathrm{d} g_{i}}{\mathrm{~d} \varepsilon} \quad, i=1,2, \ldots, n .
\end{aligned}
$$

Recall that the criterion in (3.2) depends only on the present value of the stress. On the other hand, the condition in (4.4) incorporates the idea of a short time history of the material through the expression $\phi\left(\varepsilon_{0}\right)-\phi\left(\varepsilon_{0}^{-}\right)$. The apparent independence of the former relation from stress history is only illusory. Because of the restrictions on the anticipated stressstrain states, it was found that this condition was satisfactory regardless of the stress history. The condition in (4.4) which is valid for all stress-strain states, makes this dependence explicit. However, the expression in (3.2) may be preferred under normal circumstances because of its simplicity.

The constitutive equation (4.2) can be modified to include the effects of strain rate dependence. Such effects were discussed in a review article by Perzyna [1] on viscoplasticity. For the sake of simplicity, only the yield stress is considered to be strain rate dependent. Thus, in (4.2) let $k=\tilde{k}(\dot{\varepsilon}), \tilde{k}: \mathscr{R} \rightarrow \mathscr{R}$ where $\dot{\varepsilon}$ denotes the strain rate. According to Perzyna [7], it is reasonable to assume, by virtue of experimental observation that,

$$
\tilde{k}(x)>\tilde{k}(y) \quad \text { if } x>y .
$$

Branches $(4.3)_{2,3}$ now become

$$
\begin{aligned}
& \sigma-\lambda \varepsilon-\tilde{k}(\dot{\varepsilon})=0, \\
& \sigma-\lambda \varepsilon+\tilde{k}(\dot{\varepsilon})=0 .
\end{aligned}
$$

Suppose $\varepsilon$ is increased from the initial state at a constant strain rate $\dot{\varepsilon}_{1}$. Then the response

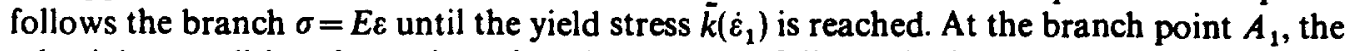
selectivity condition determines that the response follows the branch given by (4.7) with $\dot{\varepsilon}=\dot{\varepsilon}_{1}$, (see curve $0 A_{1} B_{1}$ of Fig. 5). If the process is repeated with strain rate $\dot{\varepsilon}_{2}>\dot{\varepsilon}_{1}$, the response function follows $0 A_{2} B_{2}$, by similar reasoning. 


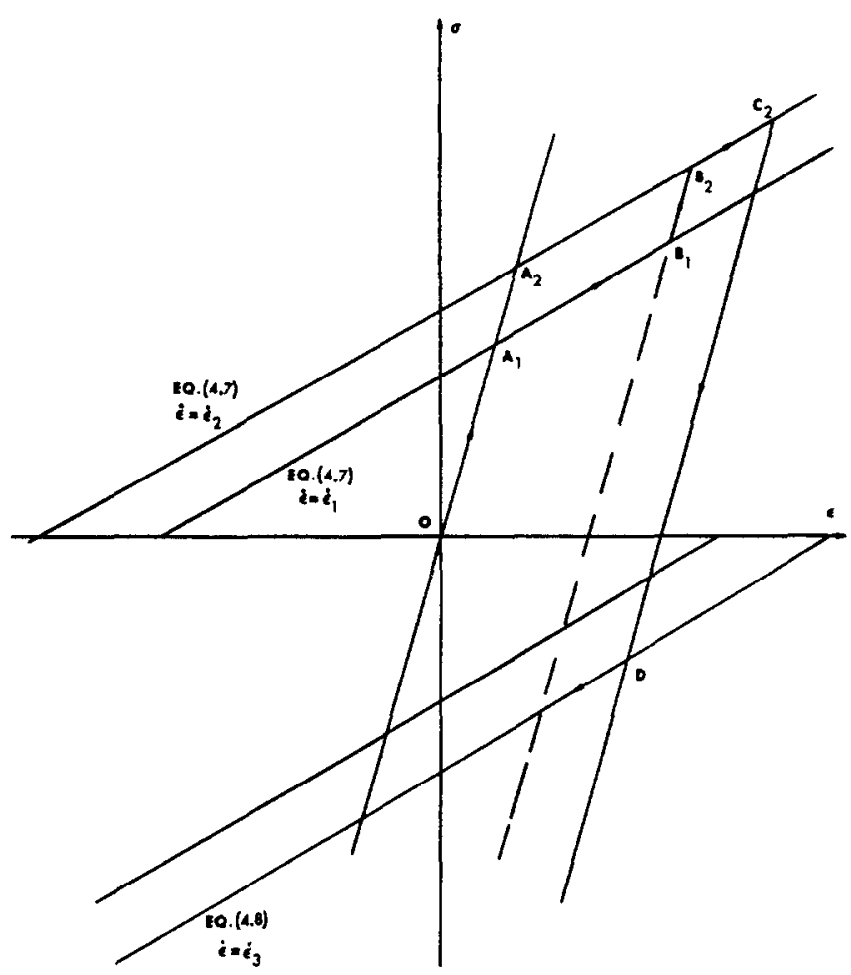

Fig. 5. Branches of equation (4.2) for the elastic-plastic model with strain rate dependence.

Let the strain be increased from the initial state at constant strain rate $\dot{\varepsilon}_{1}$ until point $B_{1}$ is reached. On changing the strain rate to $\dot{\varepsilon}_{2}>\dot{\varepsilon}_{1},(4.7)$ with $\dot{\varepsilon}=\dot{\varepsilon}_{1}$ is no longer a solution to (4.2). The response curve must then proceed along the solution to (4.3) $)_{1}$ through point $B_{1}$ until point $B_{2}$ is reached. This is a branch point of the current branch and of (4.7) with $\dot{\varepsilon}=\dot{\varepsilon}_{2}$. By the selectivity condition this latter branch is followed until $\dot{\varepsilon}$ is changed again. If loading is reversed so that $\dot{\varepsilon}=\dot{\varepsilon}_{3}<0$ at $C_{2}$, the response must follow the appropriate solution to (4.3) $)_{1}$ until point $D_{3}$ is reached. This is a branch point of the elastic unloading curve and of (4.8) with $\dot{\varepsilon}=\dot{\varepsilon}_{3}$, which is now followed.

\section{CONCLUSION}

It is seen that an implicit form of the constitutive equation, based on the notions of branching and selectivity, can be used to unify the equations which describe the response of an elastic-plastic material. In the case of elastic materials, one might consider the more elegant constitutive form

$$
A u-\lambda(\varepsilon) u=0,
$$

where the ideas of branching theory can be more gainfully employed. This, and an extended range of applications of the implicit form of constitutive equations will follow shortly.

\section{REFERENCES}

1. L. J. Zapas, Private communication.

2. J. L. Ericksen, Equilibrium of bars, J. Elasticity 5, 191 (1975).

3. A. J. A. Morgan, Some properties of media by constitutive equations in implicit form, Int. J. Engng Sci. 4, 155 (1966).

4. B. Bernstein, A unified theory of elasticity and plasticity Int. J. Engng Sci. 15, 645 (1977).

5. I. Stakgold, Branching of solutions of non-linear equations, SIAM Review 13, 289 (1971).

6. W. Ramberg and W. R. Osgood, Description of Stress-Strain Curves by Three Parameters, NACA Technical Note No. 902 , July 1943.

7. P. Perzyna, Fundamental problems in viscoplasticity, Adv. Appl. Mech. 9, 243119661 


\section{Resume}

Les materiaux, tels les elastiques-plastiques, qui montrent des regimes de reponse distincts sont habituellement representes par des equations fondamentales differentes dans chaque regime. Le present article explore une methode pour construire une equation fondamentale unifiee a partir de ces relations independantes. L'idee principale est d'ecrire cette equation unifee sous une forme implicite qui comporte comme solutions multiples ces relations independantes. On choisit cette forme de maniere a utiliser les notions de noeuds et de branches. Les differentes solutions, correspondant aux equations fondamentales pour differents regimes de reponse, peuvent alors etre considerees comme des

branches de la reponse fondamentale aux noeuds de ramification. Le choix de la branche appropriee a un noeud est regi par une condition de selection qui depend de la nature de la reponse consideree. On fournit un exemple detaille pour une reponse elastique-plastique avec et sans l'effet de dependance du taux de deformation.

\section{Zusammenfassung:}

Stoff, wie z.B. elastisch-plastische, die deutlich verschiedene Verhaltensbereiche aufweisen, werden gewohnlich mit einer verschiednen Bestimmungsgleichung fur jeden Bereich beschrieben. In dieser Arbeit wird eine Methode zur Aufstellung einer einheitlichen Bestimmungsgleichung von den verschiedenen Beziehungen untersucht. Der Hauptgedanke besteht darin, diese einheitliche Gleichung in impliziter form anzuschreiben wobe $i$ diese verschieden Losungen als nichteindeutige Losungen enthalten sind. Diese Form wird gewahlt, um den Bergriff der Verzweigungen und Verzweigungspunkte zu benutzen. Verschiedene Losungen, die den Bestimmungsgleichungen der verschiedenen Verhaltensbereiche entsprechen, werden dann als Abzweigung vom Grundverhalten an den Verzweigungspunkten betrachtet. Die Auswahl der entsprechenden Abzweigung an einem Verzweigungspunk.t wird durch ein Auswahlkriterion bestimmt, das von der Art des betrachteten Verhaltens abhangt. Ein ausfuhrliches Beispiel fur elastisch-plastisches Verhalten wird angegchen mit und ohne Berucksichtigung des Einflusses der Dehnungsgeschwindigkeit. 